\title{
A Computational Method for Sensitivity Analysis under Uncertainty
}

\author{
Hongchun Wang \\ Department of Mathematics \\ Chongqing Normal University \\ Chongqing,China \\ E-mail: springwhch@126.com
}

\begin{abstract}
Sensitivity analysis (SA) is an important part in engineering design under the uncertainty to provide valuable information about the probabilistic characteristics of a response. In this paper, the variance-based methods and the cumulative distribution function (CDF)-based sensitivity coefficients were used in sensitivity analysis. The combination of sparse grid stochastic collocation (SC) and the generalized polynomial chaos (gPC) are proposed as a method to perform the sensitivity analysis. The computational method employs the gPC as a high-order representation for random quantities, a stochastic collocation (SC) approach to deal with complex/implicit response functions, and sparse grid to use a reduced set of samples. It can reduce the computational cost associated with uncertainty assessment without much sacrifice on the optimum solution. The effectiveness is demonstrated in two numerical examples.
\end{abstract}

Keywords- Generalized Polynomial Chaos; Sensitivity Analysis; Stochastic Collocation; Sensitivity Coefficient; Uncertainty

\section{INTRODUCTION}

The traditional sensitivity analysis problem is a stage when a system is designed to find the rate of change in a model output due to change in the model input. Such an analysis is an important part in the assessment of the safety and reliability of structures, it has been widely applied in engineering design to test the validity of the assumptions made, to explore the model response behavior, to evaluate the accuracy of a model, etc., which has received increasing attention and widely acknowledged in a variety of engineer design and product development process.

Various sensitivity analysis methods, especially probabilistic sensitivity analysis (PSA) methods exist in the literature. However, a major challenge in engineering applications lies in the fact that high computational costs have to be faced. For example, one of the most commonly used methods is Monte Carlo methods or one of its variants. Although Monte Carlo methods are straightforward to apply, as they are low-order methods that provide high-fidelity results only at (often) prohibitively large computational costs, typically a large number of executions need, for the statistics converge relatively slowly. The need for large number of realizations for accurate results can incur excessive computational burden. For example, the mean value typically converges as $1 / \sqrt{K}$, where $K$ is the number of realizations (e.g.[1]). This high degree of difficulty in obtaining accurate stochastic solutions has hindered the applications of reliability-based design to a wide variety of practical engineering problems.

In this paper, Sobol' indices [2] and Wu's sensitivity coefficients [3] as the indices of sensitivity analysis are employed. Sobol' indices are one kind of variance-based methods and Wu's sensitivity coefficients are one kind of the cumulative distribution function (CDF)-based sensitivity coefficients. The generalized polynomial chaos (gPC)-based work on stochastic computations to sensitivity analysis is extended. As an alternative approach, it is able to produce accurate stochastic solutions at much reduced computational costs--in many cases several orders faster than classical sampling methods. The sparse grid stochastic collocation (SC) method [4] is also demonstrated particularly useful in evaluating various statistics, e.g. mean and variance, of system responses with high accuracy and therefore can greatly speed up the overall computations of sensitivity analysis.

\section{METHODS FOR SENSITIVITY ANALYSIS}

In general, the sensitivity analysis of system (the performance function or the transfer function) can be defined as

$$
Y=Y(X, Z)
$$

here $X=\left(x_{1}, x_{2}, \cdots, x_{d}\right) \in R^{d}$ is the design variable vector and $Z=\left(z_{1}, z_{2}, \cdots, z_{m}\right) \in R^{m}$ is a input random variable vector (also referred to as parameter vector) vector), $Y$ is an output or response variable representing product or system performance.

The goal of sensitivity analysis is to take into account the impact of uncertainties in design variables and input parameters on the probabilistic characteristic of the system (or the design performance). The word impact has different meanings under different design scenarios. For robust design, the goal of PSA is to identify those random variables which contribute the most to the variance of a response. In other words, if reducing the uncertainties in these random variables, the variance of a response could be reduced at the most. For reliability-based design, it means the contribution of reducing different sources of uncertainty to the improvement of the probability of a design constraint satisfaction. That is to say, the objective is to identify those random inputs which have the most 
influence on the probability of meeting a pre-specified target. This is particularly important if a target could not be satisfied with a required probability level.

\section{A. Sobol' indices - Variance-Based Methods}

Sobol' method [2] for variance estimation is based on an ANOVA-like decomposition of a function with an increasing dimensionality, is a variance-based method. Variance-based methods are derived from the decomposition of the total variance of a model response to different variation sources and their combinations.

From Equation (1), supposed the variance of $Y$ is $V$, the sensitivity analysis are based on the decomposition of $V$, i.e.

$$
V=V_{i}+\sum_{i<j} V_{i j}+\cdots+V_{1,2, \cdots, m}
$$

here $V_{i}$ represent the partial variance in the response due to the individual effect of a random variable $z_{i}$, the higher order terms show the interaction effects between two or more random variables.

The decomposition induces two important concepts: the main effect and the total effect. The main effect index can be shown in Equation (3):

$$
S_{i}=V_{i} / V \quad 1 \leq i \leq m
$$

The main effect index of a random variable $z_{i}$ is obtained by the normalization of the main effect variance over the total variance $Y$.

There $V_{i}$ is the partial variance in the response to the individual effect of a random variable, so the sensitivity index for the interaction between two random variables $z_{i}$ and $z_{j}$ is

$$
S_{i j}=V_{i j} / V \quad 1 \leq i<j \leq m
$$

The general sensitivity index is shown as follows

$$
S_{i, i+1 \cdots, m}=V_{i, i+1 \cdots, m} / V
$$

The total effect of a random variable $z_{i}$ can be presented

$$
S_{T_{i}}=1-S_{-i}
$$

It includes $z_{i}$ 's main effect and the interaction effect involving $z_{i}$. Where $S_{-i}=S_{1,2, \cdots, i-1, i+1, \cdots, m}$ is the index for the combined effect of all random variables except $z_{i}$.

Obviously, the variance-based methods can be directly applied to PSA in robust design as they matches with the objective of minimizing the response variance in robust design.

\section{B. CDF-based sensitivity coefficients}

$\mathrm{Wu}$ [3] derived the so-called CDF-based sensitivity coefficients for the probability, it discusses the rate of change in a probabilistic characteristic of a response $Y$ due to the changes in the probabilistic characteristics of a random input $z_{i}$, such as $\partial \mu_{Y} / \partial \mu_{z_{i}}$ and $\partial \mu_{Y} / \partial \sigma_{z_{i}}$ [5].
In general, with respect to a distribution parameter $\theta$ can be evaluated from

$$
\frac{\partial p}{\partial \theta}=\int_{\Omega} \ldots \int_{\frac{\partial f_{z}}{\partial \theta}} d x
$$

where $p$ denotes sensitivity, $f$ is the joint probability density function of all random variables , $\theta$ is the distribution parameter, $\Omega$ denotes that the expected value is evaluated using the joint probability density function (PDF) in the region. [3]

Therefore, the sensitivity coefficients can be defined as

$$
\frac{\partial p / p}{\partial \theta / \theta}=\int_{\Omega} \cdots \int \frac{\theta}{p} \frac{\partial f_{z}}{f_{z} \partial \theta} f_{z} d x=E\left[\frac{\theta \partial f_{z}}{f_{z} \partial \theta}\right]_{\Omega} \text { or } \quad \frac{\partial \ln p}{\partial \ln \theta}=E\left[\frac{\theta \partial f_{z}}{f_{z} \partial \theta}\right]_{\Omega}
$$

Based independent normal variables, Equation (6) can be simplified as

$$
\begin{gathered}
S_{\mu_{i}}=\frac{\partial p / p}{\partial \mu_{i} / \sigma_{i}}=\int_{\Omega} \ldots \int \frac{\sigma_{i}}{p} \frac{\partial \phi(u)}{\partial \mu_{i}} d u \\
=\int_{\Omega} \ldots \int u_{i}\left(\frac{\partial \phi(u)}{p}\right) d u=E\left[u_{i}\right]_{\Omega} \\
S_{\sigma_{i}}=\frac{\partial p / p}{\partial \sigma_{i} / \sigma_{i}}=\int_{\Omega} \ldots \int \frac{\sigma_{i}}{p} \frac{\partial \phi(u)}{\partial \sigma_{i}} d u \\
=\int_{\Omega} \ldots \int\left(u_{i}{ }^{2}-1\right)\left(\frac{\phi(u)}{p}\right) d u=E\left[u_{i}{ }^{2}\right]_{\Omega}-1
\end{gathered}
$$

which is the mean sensitivity coefficient and the standard deviation sensitivity coefficient[3], respectively, in which $\mu_{i}$ and $\sigma_{i}$ are the mean and the standard deviation, of the random variable $z_{i}$, the use of the standard deviation as a scale factor implies that the allowable design range of a mean value is limited to a local region characterized by the random variable variability, where E[.] is the expectation operator, $u$ is a vector of standard normal random variables transformed from $Z, \phi$ is the joint PDF of $u$. If $Z$ follows independent normal distributions, then the transformation is simply

$$
u_{i}=\frac{z_{i}-\mu_{i}}{\sigma_{i}}
$$

Otherwise, when the random variables $z_{i}$ 's are nonnormal and mutually dependent, it can be transformed to mutually independent normal variables $u_{i}$, using the following transformation [6]

$$
\begin{aligned}
u_{1} & =\Phi^{-1}\left[F_{1}\left(z_{1}\right)\right] \\
u_{i} & =\Phi^{-1}\left[F_{i}\left(z_{i} \mid z_{1}, z_{2}, \cdots, z_{i-1}\right)\right] \quad i=2,3, \cdots, m
\end{aligned}
$$

where $F_{i}$ is the original cumulative density function (CDF) of random variable $z_{i}, \Phi^{-1}$ is the inverse standardized normal CDF, and $u_{i}$ 's are the standard 
normal random variables with zero mean and unit standard deviation.

\section{FAST METHODS FOR SENSITIVITY ANALYSIS}

\section{A. Generalized Polynomial Chaos}

Generalized polynomial chaos (gPC) [7], a generalization of the classical polynomial chaos [, are able to produce accurate stochastic solutions at much reduced computational costs - in many cases several orders faster than classical sampling methods. Compared to perturbation methods, gPC offers convergence even for large levels of uncertainty and is more robust and accurate. This is an extension of the seminal work of Ghanem and Spanos on the classical polynomial chaos where only Hermite polynomials are used. Such kinds of orthogonal polynomial expansions exhibit fast convergence under sufficient conditions [7].

Generalized polynomial chaos (gPC) is a polynomial representation/approximation of random variables and processes in random space, to seek approximate a random function via orthogonal polynomials of random variables essentially.

For a performance function $Y(X, Z)$, a finite order, $P$ th-order, gPC approximation is

$$
Y_{m}^{P}(X, Z)=\sum_{i=1}^{M} \hat{Y}_{i}(X) \Phi_{i}(Z), \quad M=\left(\begin{array}{c}
m+P \\
m
\end{array}\right)
$$

Here $\left\{\Phi_{i}(Z)\right\}$ is a set of $m$-variate (the same as the length of the random variable vector $Z$ ) orthogonal polynomials of total degree up to $P$, and the total number of basis functions is $M=\left(\begin{array}{c}m+P \\ m\end{array}\right)$, and it satisfying the orthogonality conditions

$$
E\left[\Phi_{i}(Z) \Phi_{j}(Z)\right]=\int \Phi_{i}(Z) \Phi_{j}(Z) f(Z) d Z=\delta_{i j}
$$

Where $\delta_{i j}$ is the Kronecker delta function and the polynomials have been normalized to be orthonormal. $f(Z)$ is the probability density function of $Z$, it serves as a role of integration weight, and it defines the type of orthogonal polynomials $\left\{\Phi_{i}(Z)\right\}$. For example, uniform distributions are associated with Legendre polynomials, and Gaussian distributions are associated with Hermite polynomials.

The Fourier coefficients in (11) are defined as $\hat{Y}_{i}(X)=\int Y(Z) \Phi_{i}(Z) f(z) d Z=E\left(Y(Z) \Phi_{i}(Z)\right) \quad i=1,2, \cdots, M$

The error of this finite-order projection can be defined as

$$
\varepsilon_{G}(X)=\left\|Y(X, Z)-Y_{m}^{P}(X, Z)\right\|_{L_{f}^{2}}
$$

and will converge to zero in the mean square sense as the order of approximation $P$ is increased, i.e.

$$
\left\|Y(X, Z)-Y_{m}^{P}(X, Z)\right\|_{L_{f}^{2}}=\left(E\left[\left(Y(X, Z)-Y_{m}^{P}(X, Z)\right)^{2}\right]\right)^{1 / 2} \rightarrow 0, \quad P \rightarrow \infty
$$

Therefore, we can use $Y_{m}^{P}(X, Z)$ as an approximation instead of $Y(X, Z)$.

\section{B. Stochastic collocation (SC)}

In practice, the definition (13) of the expansion coefficients is not useful as the response function $Y(X, Z)$ is usually unknown. One then needs to construct numerical procedures to solve for the coefficients approximately.

Therefore, we again seek an approximate solution to the observable $Z$ in the form of gPC expansion in stochastic collocational approach

$$
\tilde{\hat{Y}}_{i}(X)=\sum_{j=1}^{Q} Y\left(Z^{(j)}\right) \Phi_{i}\left(Z^{(j)}\right) \alpha^{(j)} \quad i=1,2, \cdots, M
$$

Here $\left\{Z^{(j)}, \alpha^{(j)}\right\}_{j=1}^{Q}$ are a set of nodes and weights, where $Z^{(j)}=\left(z_{1}^{j}, z_{2}^{j}, \cdots, z_{m}^{j}\right)$ and $\alpha^{(j)}$ denote the $j$-th node and its associated weights, $Q$ is the number of nodes. With such a choice of the nodal set, (16) approximates (13) approaches zero as $Q \rightarrow \infty$.

\section{Choices of nodal set}

The choice of the nodal set is the key issue in SC method in order to make (16) accurate and efficient, it is essential that the nodal set is a good cubature rule such that multiple integrals can be well approximated by a weighted discrete sum in the form of (13). Many choices are available in one-dimensional $(m=1)$ space, for sufficiently smooth integrand, one of the optimal choices is quadrature rules based on orthogonal polynomials $\left\{\Phi_{i}(Z)\right\}$ in (12), it is usually the Gauss quadratures [8]. The challenge is in multi-dimensional spaces with $m>1$, especially for large dimensions $m>>1$.

In this paper, the Smolyak sparse grid is employed to use the sensitivity analysis for system, because it offers a systematic way of increasing accuracy, and therefore can achieve highly accurate results.

Other choices of nodal set can be sought for (16), those obtained by cubature rules or design of experiments, are also viable. For example, one can employ Monte Carlo method, where the nodes are randomly generated and $\alpha^{(j)} \equiv 1 / Q \quad j=1, \cdots, Q$. However, the (relatively) larger statistical errors due to the (relatively) slower convergence of Monte Carlo method may incur large aliasing errors.

Subsequently, an approximation of the true gPC expansion can be express as

$$
\tilde{Y}_{m}^{P}(X, Z)=\sum_{i=1}^{M} \tilde{\hat{Y}}_{i}(X) \Phi_{i}(Z), \quad M=\left(\begin{array}{c}
m+P \\
m
\end{array}\right)
$$

In other word, (17) is an approximation of the exact gPC expansion (11), and the overall mean-square error can be bounded by

$$
\varepsilon \equiv\left(\left[\int Y(X, Z)-\tilde{Y}_{m}^{P}(X, Z)\right]^{2} f(Z) d Z\right)^{\frac{1}{2}}
$$

The error be controlled in practice, and can be refined by increasing the order of the gPC expansion, and the accuracy of the integration rule, etc. For more detailed discussions see [10].

\section{D. $\quad$ gPC-based sensitivity analysis}

When combined sparse grid stochastic collocation approach with gPC in a polynomial form of (17), and various post-processing procedures can be carried out to 
obtain statistics, efficient numerical methods can be readily constructed for complex engineering systems with uncertainty.

The mean value is the first expansion coefficient $\tilde{\hat{Y}}_{1}$, i.e.

$$
E[Y]=E[Y(X, Z)] \approx \int \sum_{i=1}^{M} \tilde{\hat{Y}}_{i}(X) \Phi_{i}(Z) \rho(Z) d Z=\tilde{\hat{Y}}_{1}(X)
$$

The variance can be evaluated as

$$
\operatorname{Var}[Y]=E\left[(Y(X, Z)-E Y(X, Z))^{2}\right] \approx \sum_{i=2}^{M} \tilde{\hat{Y}}_{i}^{2}(X)
$$

where the first mode (the mean mode) $\tilde{\hat{Y}}_{1}$ is excluded from the summation [11].

Thus, the Sobol' indices can be evaluated when equation (18) and (19) are employed, and the Wu's sensitivity coefficients of CDF-based can be approximated conveniently by using (20)

$$
\frac{\partial Y}{\partial z_{i}} \approx \sum_{i=1}^{M} \tilde{\hat{Y}}_{i}(X) \frac{\partial \Phi_{i}(Z)}{\partial z_{i}}, \quad i=1,2, \cdots, m
$$

where the terms $\partial \Phi_{i}(Z) / \partial z_{i}$ are the derivatives of the polynomials and can be calculated conveniently. With the sparse grids SC-based are used and the sensitivity derivatives available, the Wu's sensitivity coefficients can be approximated.

In summary, when the design variable vector $X$ is known, the sensitivity analysis procedure consists of the following steps:

Step 1: Determine the basis function $\Phi(Z)$ type according to the distribution of $Z$.

Step2: Choose a collocation nodal set $\left\{Z^{(j)}, \alpha^{(j)}\right\}_{j=1}^{Q}$ for the random vector $Z$.

Step3: Evaluate the performance function $Y\left(Z^{(j)}\right)=Y\left(X, Z^{(j)}\right)$.

Step4: Evaluate the approximate gPC expansion coefficients via Equation (16).

Step5: Compute sensitivity coefficients through equation (18), (19), (20).

Step6: Determine the sensitivity of each random variable according to the value of sensitivity coefficients.

\section{NUMERICAL EXAMPLES}

\section{A. A Mathematical Example}

Consider a simple nonlinear model for a performance function as follows

$$
Y=\sin \left(z_{1}+1\right) e^{z_{2}} / \ln \left(\frac{1}{2}+z_{3}^{3}\right)
$$

where $Z=\left(z_{1}, z_{2}, z_{3}\right)$ are three independent Gaussian random variables with zero mean and standard deviation 1 , i.e. $z_{i} \sim N(0,1) \quad i=1,2,3$.

From equation (19), we can evaluate the variance $\operatorname{Var}[Y] \approx \sum_{i=2}^{M} \tilde{\hat{Y}}_{i}^{2}(X)$ of $Y$ to use sparse grids SC-based. When $z_{i} \quad i=1,2,3$ is fixed its mean value respectively, the variance of different variation sources and their combinations can be evaluated. Therefore, the main effect indices and total effect indices of a random variable is computed that shown in Table 1, where the orthogonal

\begin{tabular}{|c|c|c|c|c|}
\hline \multirow[t]{2}{*}{ Method } & \multirow{2}{*}{$\begin{array}{l}\text { Number of } \\
\text { realizations }\end{array}$} & \multirow{2}{*}{$\begin{array}{l}\text { Random } \\
\text { Variable }\end{array}$} & \multicolumn{2}{|c|}{ Effect Indices } \\
\hline & & & main effect & total effect \\
\hline \multirow[t]{3}{*}{$\begin{array}{l}\text { This paper's } \\
\text { method }\end{array}$} & \multirow[t]{3}{*}{25} & $z_{1}$ & $\begin{array}{c}5.6296 \mathrm{e}- \\
007\end{array}$ & 0.4071 \\
\hline & & $z_{2}$ & $\begin{array}{c}3.8023 \mathrm{e}- \\
006\end{array}$ & 0.7837 \\
\hline & & $z_{3}$ & 0.0177 & 0.9836 \\
\hline \multirow[t]{3}{*}{ Monte Carlo } & \multirow[t]{3}{*}{100000} & $z_{1}$ & $\begin{array}{c}5.5099 \mathrm{e}- \\
007\end{array}$ & 0.4121 \\
\hline & & $z_{2}$ & $\begin{array}{c}8.0354 \mathrm{e}- \\
006\end{array}$ & 0.7561 \\
\hline & & $z_{3}$ & 0.0183 & 0.9982 \\
\hline
\end{tabular}
polynomials of total degree up to 2 , total number of nodes SC-based sparse grid is 25 .

TABLE I. COMPUTATION RESULT

From Table 1, it is noted that the random parameter $z_{3}$ is the most critical. In other word, $z_{3}$ is the most sensitive, there is a greatest impact on the system that by reducing or increasing the same amount of variance $z_{3}$ leads to the largest reliability increase or reduce than any other variables, we need control it carefully in solving practical problems.

We define the error for the effect index

$$
\varepsilon=\left|\frac{S_{i}-S_{M C i}}{S_{M C i}}\right|
$$

where $S_{i}$ is $z_{i}$ 's effect index through this paper's method, $S_{M C i}$ is $z_{i}$ 's effect index through Monte Carlo simulation method, we can calculate the error for the effect index are lower than $4 \%$.

With the result of Table 1, we can see this paper's method has almost the same precision with Monte Carlo simulation, but it much faster than Monte Carlo simulation. For example, the CPU time of $z_{3}$ 's main effect is 0.46 seconds, and its the CPU time is 9.31 seconds with Monte Carlo simulation. Here, the CPU time based on Intel Core Duo CPU T6600, installed memory 4.00GB, 64-bit Operating System.

\section{B. A Cantilever Beam Example}

In this example, a cantilever beam in vertical and lateral bending [11] is used (see Fig. 1). The beam is loaded at its tip by the vertical and lateral loads $Y$ and $Z$, respectively. Its length $\mathrm{L}$ is equal to $100 \mathrm{in}$. The width $w$ and thickness $t$ of the cross-section are random design variables.
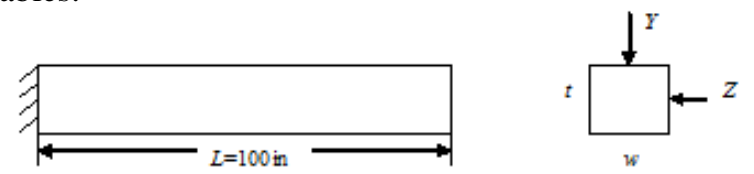

Figure 1. Cantilever beam under vertical and lateral bending 
One non-linear failure mode is used representing yielding at the fixed end of the cantilever. $\quad G(y, Z, Y, w, t)=y-\left(\frac{600}{w t^{2}} * Y+\frac{600}{w^{2} t} * Z\right)$ represents the failure mode.

The random design variables $w$ and $t$ are normally distributed with $\sigma_{w}=\sigma_{t}=0.225 . Y, Z$ and $y$ are normally distributed random parameters with $Y \sim N(1000,100) \quad \mathrm{lb}, \quad Z \sim N(500,100) \quad \mathrm{lb}$, $y \sim N(40000,2000) \mathrm{psi} ; y$ is the random yield strength, $Y$ and $Z$ are mutually independent random loads in the vertical and lateral directions respectively.

Table 2 shows the CDF-based sensitivity coefficients of each random variable.

TABLE II. CDF-BASED SENSITIVITY COEFFICIENTS OF RANDOM VARIABLES

\begin{tabular}{|c|c|c|c|c|c|}
\hline & $w$ & $t$ & $Y$ & $Z$ & $y$ \\
\hline \multirow{3}{*}{$S_{\mu}$} & $4.416953 \mathrm{e}-$ & -3.20860 & $-1.369552 \mathrm{e}-$ & 4.01655 & -9.16878 \\
& 003 & $9 \mathrm{e}-003$ & 002 & $7 \mathrm{e}-002$ & $0 \mathrm{e}-004$ \\
& & & & & \\
\hline \multirow{2}{*}{$S_{\sigma}$} & -1.15936 & -4.14454 & $9.897566 \mathrm{e}+00$ & 9.91743 & 3.686898 \\
& $8 \mathrm{e}-003$ & $8 \mathrm{e}-003$ & 1 & $8 \mathrm{e}+001$ & $\mathrm{e}-003$ \\
& & & & & \\
\hline
\end{tabular}

It is observed that the importance rankings of random variables for each CDF-based sensitivity coefficients from Table 2. For example, random variables $Y, Z$ are more sensitive than others are. From Fig. 2, we can see the same result. Fig. 2 shows the relationship of each random variable variance reduction and the sensitivity improvement. It is confirmed in Fig. 2 that by reducing the same amount of variance, $Y, Z$ leads to the largest sensitivity increase than any other variables.

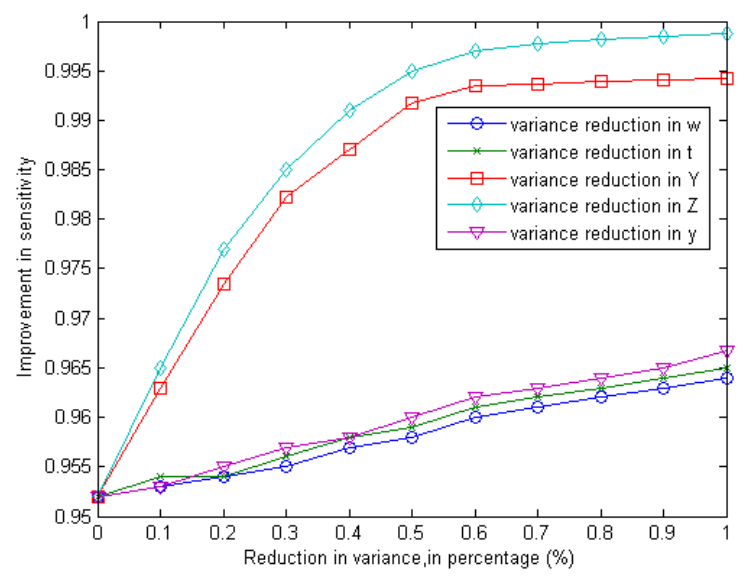

Figure 2. The relation of improvement sensitivity and variance reduction

\section{CONCLUSION}

Sensitivity analysis is a useful tool in design under uncertainty by providing valuable information of the impact of uncertainty sources. It can be used to identify those insignificant variables and to reduce the design problem dimension. In reliability-based design, some design parameters can be adjusted. These parameters might be deterministic, such as tightly controlled geometries. Reliability improvements could be achieved by a combination of various design modifications such as tightening tolerances, using better materials, or changing geometries. A decision analysis based on the cost, manufacturing, and other design considerations may be performed to select an optimal design. Upon a design change, a reanalysis should generally be conducted to confirm the improved reliability.

In this paper, a computationally efficient and fast method for sensitivity analysis, based on generalized polynomial chaos representation for random quantities and a stochastic collocation (SC) approach choice of the nodal set, has been presented. The effectiveness and feasibility of the method is demonstrated by two numerical examples.

\section{ACKNOWLEDGMENT}

This research was supported by a grant from the National Social Science Foundation of China (Grant No. 13BTJ008).

\section{REFERENCES}

[1] G.S. Fishman, Monte Carlo: Concepts, Algorithms, and Applications, Springer-Verlag, New York, 1996.

[2] I. M.Sobol, Global Sensitivity Indices for Nonlinear Mathematical Models and Their Monte Carlo Estimates, Mathematics and Computers in Simulation, 55(2001) 271-280.

[3] Y.-T.Wu, Computational Methods for Efficient Structural Reliability and Reliability Sensitivity Analysis, AIAA Journal, 32(1994) 1717-1723.

[4] D. Xiu and J.S. Hesthaven, High Order Collocation Methods for Differential Equations with Random Inputs, SIAM J. Sci. Comput., 27(2005), 1118-1139.

[5] N.A.Eisenberg, B.Sagar, Importance measures for nuclear waste repositories, Rel Engng Saf, 70(2000)217-239

[6] M. Rosenblart, Remarks on a multivariate transformation, The Annals Math Statist, 23(1952) 470-472.

[7] D. Xiu and G.E. Karniadakis, The Wiener-Askey polynomial chaos for stochastic differential equations, SIAM J. Sci. Comput., 24(2002) 619-644.

[8] Gene H. Golub and John H. Welsch, Calculation of Gauss Quadrature Rules, Mathematics of Computation 23 (1969): 221 230.

[9] D. Xiu, Efficient collocational approach for parametric uncertainty analysis, Commun. Comput. Phys., 2(2007) 293-309.

[10] J. Li and D. Xiu, Surrogate Based Method for Evaluation of Failure Probability Under Multiple Constraints, SIAM J. Sci. Comput., 36((2014)), A828-A845.

[11] Y.-T. Wu, Y.Shin, R.Sues and M.Cesare, Safety - Factor Based Approach for Probabilistic - Based Design Optimization, 42nd AIAA/ASME/ASCE/AHS/ASC Structures, Structural Dynamics and Materials Conference, Seattle, WA, 2001. 